Tohoku Math. J.

53 (2001), 593-615

\title{
BIRKHOFF DECOMPOSITIONS AND IWASAWA DECOMPOSITIONS FOR LOOP GROUPS
}

\author{
VLADIMIR BALAN $^{\dagger}$ AND JOSEF DORFMEISTER*
}

(Received February 9, 2000, revised December 25, 2000)

\begin{abstract}
Representations of arbitrary real or complex invertible matrices as products of matrices of special type have been used for many purposes. The matrix form of the Gram-Schmidt orthonormalization procedure and the Gauss elimination process are instances of such matrix factorizations. For arbitrary, finite-dimensional, semisimple Lie groups, the corresponding matrix factorizations are known as Iwasawa decomposition and Bruhat decomposition. The work of Matsuki and Rossmann has generalized the Iwasawa decomposition for the finite-dimensional, semisimple Lie groups. In infinite dimensions, for affine loop groups/Kac-Moody groups, the Bruhat decomposition has an, also classical, competitor, the Birkhoff decomposition. Both decompositions (in infinite dimensions), the Iwasawa decomposition and the Birkhoff decomposition, have had important applications to analysis, e.g., to the Riemann-Hilbert problem, and to geometry, like to the construction of harmonic maps from Riemann surfaces to compact symmetric spaces and compact Lie groups. The Matsuki/Rossmann decomposition has been generalized only very recently to untwisted affine loop groups by Kellersch and facilitates the discussion of harmonic maps from Riemann surfaces to semisimple symmetric spaces.

In the present paper we extend the decompositions of Kellersch and Birkhoff for untwisted affine loop groups to general Lie groups. These generalized decompositions have already been used in the discussion of harmonic maps from Riemann surfaces to arbitrary loop groups [2].
\end{abstract}

1. Introduction. In recent years, loop groups have been used to investigate families of geometric objects. Uhlenbeck's investigation of harmonic maps from surfaces to compact Lie groups [24] represents the most outstanding example for this. Later, the study of surfaces of constant mean curvature (the Gauss map of which is harmonic with values in the homogeneous space $\left.S^{2}=S U(2) / U(1)\right)$ lead to a generalized Weierstrass representation for harmonic maps from surfaces to compact symmetric spaces [10]. By now, similar procedures have been employed to investigate geometric objects like Willmore surfaces [15] and affine spheres [7]. In these two cases homogeneous spaces occur, for which the transitive group is not compact. We would expect that in many examples such a situation will occur. We therefore feel that it will be useful to establish, for general Lie groups and general homogeneous spaces, the basic properties for loop groups, as needed for the papers mentioned above. The most salient

2000 Mathematics Subject Classification. Primary 22E67; Secondary 22E15, 22E25, 22 E46.

* Work partially supported by NSF Grant DMS-9705479 and Sonderforschungsbereich 288, Differentialgeometrie und Quantenphysik, TU-Berlin.

$\dagger$ Work supported by Fulbright Grant \#21945-5/13/97. 
features of loop groups used in [24], [10], [14], [7] are certainly generalized Birkhoff and Iwasawa decompositions for loop groups. In this note we therefore establish these generalized decomposition theorems for loop groups associated with arbitrary Lie groups and illustrate the results by several typical examples. We would like to point out that the Iwasawa decomposition in the case of the loop group associated with a general semisimple Lie group has been established by Kellersch in his dissertation [20]. In subsequent papers [2], [3], we plan to generalize the method used in [24] and [10] to investigate harmonic maps into general Lie groups and harmonic maps into generalized symmetric spaces. This paper deals exclusively with untwisted loop groups. We plan to discuss the twisted case elsewhere.

2. Preliminaries. Let $G$ be a connected real analytic Lie group which admits a faithful finite dimensional continuous representation. Then by the Levi theorem [17, Theorem 18.4.3] there exist a reductive, analytic subgroup $H$ of $G$, and a simply connected, solvable, analytic subgroup $B$ of $G$, normal in $G$, such that $G$ can be written as a semidirect product

$$
\begin{aligned}
& G=H \ltimes B, \\
& B \cong A \ltimes N,
\end{aligned}
$$

where $A$ is a simply connected, connected abelian Lie group and $N$ is a simply connected and connected unipotent Lie group with Lie algebra $\operatorname{Lie}(N)=[\operatorname{Lie}(B), \operatorname{Lie}(B)]$.

In particular, $N$ is a normal subgroup of $B$ and we have

$$
A \cong \boldsymbol{R}^{p} .
$$

The complexified groups [17, 17.5 ], $B^{C}$ and $H^{C}$ inherit the properties of $B$ and $H$. Hence $H^{C}$ is a reductive complex group, and $B^{C}$ is also a simply connected, solvable normal subgroup of $G^{C}$. Then $G^{C}$ admits a faithful finite-dimensional complex representation and satisfies [17,

$$
G^{C}=H^{C} \ltimes B^{C} .
$$

Being complex and reductive, $H^{C}$ is the complexification of a (maximal) real compact subgroup $K$ of $H^{C}$, that is, $H^{C}=K^{C}$. On the other hand, being solvable and simply connected, the group $B^{C}$ decomposes canonically

$$
{ }_{B}^{C}=A^{C} \cdot N^{C},
$$

where $A^{C}$ is abelian and $N^{C}=\left[B^{C}, B^{C}\right]$ is simply connected and is the nilradical of $B^{C}$ [5, Chap. 1, §5.3, Theorem 1]. $N^{C}$ is closed in $B^{C}$ and has the Lie algebra $\operatorname{Lie}\left(N^{C}\right)=$ $\left[\operatorname{Lie}\left(B^{C}\right), \operatorname{Lie}\left(B^{C}\right)\right]$. We remark that by [17, Theorem 17.4.1] we have

$$
{ }_{B}^{C} / N^{C} \cong A^{C} .
$$

Also we obtain

$$
{ }_{A} \boldsymbol{C} \cong \boldsymbol{C}^{p} .
$$




\section{Loop groups.}

3.1. Let $\rho$ be a finite-dimensional faithful representation of $G^{C}$ and for $g: S^{1} \rightarrow G^{C}$ continuous, let $\tilde{g}=\sum_{k \in \boldsymbol{Z}} a_{k} \lambda^{k}, a_{k} \in \rho\left(G^{\boldsymbol{C}}\right), \lambda \in S^{1}$, be the associated Fourier series. Then the loop group $\Lambda G^{C}$ defined by

$$
\Lambda G^{C}=\left\{g: S^{1} \rightarrow G^{C} \mid \tilde{g} \text { is absolutely convergent }\right\}
$$

is a complex Banach Lie group with the Wiener topology ([19, Chap. I, 6.1], [8, §1]). We shall also use its subgroups

$$
\begin{aligned}
& \Lambda G=\left\{g \in \Lambda G^{C} \mid g(\lambda) \in G \text { for all } \lambda \in S^{1}\right\}, \\
& \Lambda^{+} G^{C}=\left\{g \in \Lambda G^{C} \mid g \text { and } g^{-1} \text { extend holomorphically to } \mathbf{D}\right\}, \\
& \Lambda^{-} G^{C}=\left\{g \in \Lambda G^{C} \mid g \text { and } g^{-1} \text { extend holomorphically to } \bar{C} \backslash \mathbf{D}\right\},
\end{aligned}
$$

where $\mathbf{D}=\{z \in \boldsymbol{C}|| z \mid<1\}$ and $\overline{\boldsymbol{C}}$ denotes the Riemann sphere. We shall also denote

$$
\Lambda_{*}^{-} G^{C}=\left\{g \in \Lambda^{-} G^{C} \mid \tilde{g}=\sum_{k \leq 0} a_{k} \lambda^{k}, a_{0}=I\right\},
$$

the meaning of the subscript "**" remaining the same when considering instead of $G$ one of its subgroups.

Remark. $\Lambda_{*}^{-} G^{C} \cap \Lambda^{+} G^{C}=\{e\}$, where $e \in G^{C}$ is the unity of the group $G^{C}$.

3.2. Any normal complex subgroup $G^{\prime \prime}$ of a simply connected complex Lie group $G^{\prime}$ is closed, and the associated bundle $\left(G^{\prime}, \pi, G^{\prime} / G^{\prime \prime}\right)$ admits an analytic section $\tau: G^{\prime} / G^{\prime \prime} \rightarrow G^{\prime}$ such that the mapping

$$
G^{\prime \prime} \times G^{\prime} / G^{\prime \prime} \rightarrow G^{\prime}, \quad\left(g^{\prime \prime}, \bar{g}\right) \mapsto g^{\prime \prime} \cdot \tau(\bar{g})
$$

is an analytic manifold isomorphism [17, Chap. 12, Theorem 1.2, p. 135]. Then, for $G^{\prime}=$ $G^{C}, G^{\prime \prime}=B^{C}$, we have the diffeomorphism $B^{C} \times\left(G^{C} / B^{C}\right) \cong G^{C}$, which at the loop group level leads to the splittings

$$
\begin{aligned}
\Lambda G^{C} & \cong \Lambda H^{C} \cdot \Lambda B^{C}, \\
\Lambda^{-} G^{C} & \cong \Lambda^{-} H^{C} \cdot \Lambda^{-} B^{C}, \\
\Lambda^{+} G^{C} & \cong \Lambda^{+} H^{C} \cdot \Lambda^{+} B^{C} .
\end{aligned}
$$

Note. Under the assumptions made in 3.1, the Wiener-Levi theorem [19, Chap. VIII, Theorem 3.10] shows that the projection $\pi$ of the bundle $\left(G^{\prime}, \pi, G^{\prime} / G^{\prime \prime}\right)$ projects loops to loops, i.e., the absolute Fourier series convergence is preserved by composition of loops $f: S^{1} \rightarrow G^{\prime}$ with the mapping $\pi$. Therefore the isomorphism $H^{C} \cong G^{C} / B^{C}$ leads to an isomorphism of the corresponding loop groups.

4. The Birkhoff decomposition. Let $N^{C}$ be the nilradical of $B^{C}$. Then $N^{C} \triangleleft G^{C}$. We assume without loss of generality that in the given representation, $N^{C}$ is represented by upper triangular matrices with units on the diagonal. 
4.1. Based on the decompositions in 2.1 we have the corresponding decompositions for loop groups

$$
\begin{aligned}
& \Lambda B^{C} \cong \Lambda A^{C} \cdot \Lambda N^{C}, \\
& \Lambda A^{C} \cong \Lambda C^{p} .
\end{aligned}
$$

Hence the decomposition of the Wiener algebra into "plus part" and "minus part" is rephrased as

$$
\Lambda A^{C} \cong \Lambda_{*}^{-} A^{C} \cdot \Lambda^{+} A^{C} .
$$

This yields a unique representation of the elements of $\Lambda A^{C}$.

Using (2.1.6) and the Wiener-Levi theorem [19, Chap. VIII, Theorem 3.10], we obtain

$$
\Lambda B^{C} \cong \Lambda^{-} A^{C} \cdot \Lambda^{+} A^{C} \cdot \Lambda N^{C} .
$$

One can even show, using the subscript notation in (3.1.1),

$$
\Lambda B^{C} \cong \Lambda_{*}^{-} A^{C} \cdot \Lambda^{+} A^{C} \cdot \Lambda N^{C} .
$$

This yields a unique representation of the elements of $\Lambda B^{C}$.

4.2. The loop group $\Lambda N^{C}$ can be decomposed as follows.

LEMMA. There exists a canonical diffeomorphism:

$$
\Lambda N^{C} \cong \Lambda_{*}^{-} N^{C} \times \Lambda^{+} N^{C} .
$$

Proof. Let $\mathbf{n}=\operatorname{Lie}(N)$ be the Lie algebra of $N$. We define

$$
\mathbf{n}^{(0)}=\mathbf{n} \quad \text { and } \quad \mathbf{n}^{(k+1)}=\left[\mathbf{n}, \mathbf{n}^{(k)}\right], \quad k \geq 0 .
$$

Let $\left\{b_{k j}\right\}_{k \geq 0, j \in\left\{1, \ldots, m_{k}\right\}}$ be a basis for $\mathbf{n}$, where for fixed $k$, the $\left\{b_{k j}\right\}_{j \in\left\{1, \ldots, m_{k}\right\}}$ form a basis of a complement of $\mathbf{n}^{(k+1)}$ in $\mathbf{n}^{(k)}$. Then every element $n \in N$ can be written in the form

$$
n=\prod_{i \geq 0} \prod_{j=0}^{m_{i}} \exp \left(\alpha_{i j} \cdot b_{i j}\right) \text {. }
$$

Let $\tilde{n} \in \Lambda N^{C}$. We define inductively

$$
\tilde{n}^{(k+1)}=u_{k}^{-} \cdot \tilde{n}^{(k)} \cdot u_{k}^{+}, \quad k \geq 0, \quad \tilde{n}^{(0)}=\tilde{n},
$$

where

$$
u_{k}^{-}=\prod_{j=0}^{m_{k}} \exp \left(-\alpha_{k j}^{(k)-} \cdot b_{k j}\right), \quad u_{k}^{+}=\prod_{j=0}^{m_{k}} \exp \left(-\alpha_{k j}^{(k)+} \cdot b_{k j}\right),
$$

and where at each step $k \geq 0$, the coefficients $\alpha_{k j}^{(k) \pm}$ are provided by the decompositions

$$
\tilde{n}^{(k)}=\prod_{i \geq k} \prod_{j=0}^{m_{i}} \exp \left(\alpha_{i j}^{(k)} \cdot b_{i j}\right), \quad \alpha_{k j}^{(k)}=\alpha_{k j}^{(k)-}+\alpha_{k j}^{(k)+} \in \Lambda^{-} \boldsymbol{C}+\Lambda^{+} \boldsymbol{C},
$$


with the constant terms contained in $\alpha_{k j}^{(k)+}$. After a finite number $m$ of steps we obtain $\tilde{n}^{(m)}=$ $e \in \Lambda N^{C}$. Hence, denoting

$$
v_{k}^{-}=\left(u_{k}^{-}\right)^{-1} \in \Lambda^{-} N^{C}, \quad v_{k}^{+}=\left(u_{k}^{+}\right)^{-1} \in \Lambda^{+} N^{C}, \quad k \in\{0, \ldots, m-1\},
$$

we infer the decomposition

$$
\tilde{n}=\left(v_{0}^{-} \ldots v_{m-1}^{-}\right) \cdot\left(v_{m-1}^{+} \ldots v_{0}^{+}\right) \in \Lambda^{-} N^{C} \cdot \Lambda^{+}{ }^{C} .
$$

The diffeomorphism between the set theoretic product (4.2.1) and (4.2.7) follows from [8, Corollary 3.1.4].

4.3. We are now able to prove the Birkhoff splitting for the solvable group $B$. Using the results of Section 4.1, the elements of $\Lambda B^{C}$ can be split also as follows.

Lemma. $\quad \Lambda B^{C}=\Lambda_{*}^{-} B^{C} \cdot \Lambda^{+} B^{C}$. More precisely, each element $b \in \Lambda B^{C}$, can be written uniquely as

$$
b=b_{-} \cdot b_{+} \in \Lambda_{*}^{-} B^{C} \cdot \Lambda^{+} B^{C},
$$

with $b \in \Lambda_{*}^{-} B^{C}$ and $b_{+} \in \Lambda^{+} B^{C}$. Moreover, $\Lambda B^{C} \cong \Lambda_{*}^{-} B^{C} \times \Lambda^{+} B^{C}$.

PROOF. Using the decompositions (4.1.1) and (4.1.4), we have

$$
b=a_{-} a_{+} \cdot n
$$

with $a_{-} \in \Lambda_{*}^{-} A^{C}, a_{+} \in \Lambda^{+} A^{C}$ and $n \in \Lambda N^{C}$. Then $b=a_{-} \tilde{n} a_{+}$, where $\tilde{n}=a_{+} n a_{+}{ }^{-1} \in$ $\Lambda N^{C}$. But, according to (4.2.1), $\tilde{n}$ has a decomposition of the form $\tilde{n}=\tilde{n}_{-} \cdot \tilde{n}_{+} \in \Lambda_{*}^{-} N^{C}$. $\Lambda^{+} N^{C}$, and hence

$$
b=a_{-} \tilde{n}_{-} \tilde{n}_{+} a_{+},
$$

with $a_{-} \in \Lambda_{*}^{-} A^{C}, a_{+} \in \Lambda^{+} A^{C}, \tilde{n}_{-} \in \Lambda_{*}^{-} N^{C}$ and $\tilde{n}_{+} \in \Lambda^{+} N^{C}$. For elements of this form all factors are determined uniquely by $b$. To verify the last statement we apply $[8$, Corollary 3.1.4].

4.4. In view of (3.2.1) it will be useful to state the Birkhoff decomposition theorem for reductive groups $[23,8.7],[8,8.6]$. With the notation of Section 2 , we have

THEOREM. Let $H$ be a real, reductive analytic group. Then

$$
\Lambda H^{C}=\Lambda^{-} H^{C} \cdot \Lambda^{d} H^{C} \cdot \Lambda^{+} H^{C},
$$

where $\Lambda^{d} H^{C}$ consists of all homomorphisms from $S^{1}$ into a maximal torus in $H^{C}$.

REMARK. The decomposition (4.4.1) for $h, h=h_{-} s h_{+}$, can be made unique if we impose additional conditions [22, Corollary 5(c)] for the semisimple part of $H^{C}$ and if we assume for the center part of $H^{C}$ that in the decomposition $c=c_{-} \lambda^{r} c_{+}$, we have, e.g., $c_{+}(\lambda=0)=1$.

For the general Birkhoff decomposition theorem 4.5 below we will need a decomposition of $\Lambda B^{C}$ relative to some $s \in \Lambda^{d} H^{C}$. For this we consider the adjoint action of $H^{C}$ on $B^{C}$ and the induced action of $\Lambda H^{C}$ on $\Lambda B^{C}$. The elements of $\Lambda^{d} H^{C}$ can then be realized [23, 5.1], [23, 8.7] as homomorphisms of $S^{1}$ into some maximal toral subgroup $C$ contained in 
$H^{C}$. Decomposing $\operatorname{Lie}\left(B^{C}\right)=\bigoplus \operatorname{Lie}\left(B^{C}\right)_{\sigma}$ into weight spaces relative to $C$, we have [18, Chap. 7.9] that $s$ acts on $\Lambda B^{C}{ }_{\sigma}$ by

$$
s v_{\sigma} s^{-1}=\lambda^{k} v_{\sigma}, \quad k=k(s, \sigma) \in \boldsymbol{Z} .
$$

Thus on the Lie algebra level we obtain

$$
\operatorname{Lie}\left(\Lambda^{-} B^{C}\right)=A_{s}^{-}+A_{s}^{+},
$$

$$
A_{s}^{-} \cap A_{s}^{+}=\{0\},
$$

where

$$
A_{s}^{-}=\left\{x \in \operatorname{Lie}\left(\Lambda^{-} B^{C}\right) \mid s x s^{-1} \in \operatorname{Lie}\left(\Lambda_{*}^{-} B^{C}\right)\right\}
$$

and

$$
A_{s}^{+}=\left\{x \in \operatorname{Lie}\left(\Lambda^{-} B^{C}\right) \mid s x s^{-1} \in \operatorname{Lie}\left(\Lambda^{+} B^{C}\right)\right\} .
$$

It is easy to verify that $A_{s}^{-}$and $A_{s}^{+}$are closed subalgebras of $\operatorname{Lie}\left(\Lambda^{-} B^{C}\right)$.

Let $\left(\Lambda^{-} B^{C}\right)_{s}^{-}$and $\left(\Lambda^{-} B^{C}\right)_{s}^{+}$denote the integral subgroups [5, Chap. 3] of $\Lambda^{-} B^{C}$ with Lie algebras $A_{s}^{-}$and $A_{s}^{+}$respectively, then $s\left(\Lambda^{-} B^{C}\right)_{s}^{-} s^{-1} \subset \Lambda_{*}^{-} B^{C}$, while $s\left(\Lambda^{-} B^{C}\right)_{s}^{+} s^{-1} \subset$ $\Lambda^{+} B^{C}$. Hence we can apply [8, Corollary 3.1.4] and obtain a) and b) of the

LEMMA. a) The groups $\left(\Lambda^{-} B^{C}\right)_{s}^{-}$and $\left(\Lambda^{-} B^{C}\right)_{s}^{+}$are Banach Lie subgroups of $\Lambda^{-} B^{C}$

b) the group product of the two groups is diffeomorphic with the set product of the two groups;

c) $\Lambda^{-} B^{C}=\left(\Lambda^{-} B^{C}\right)_{s}^{-} \cdot\left(\Lambda^{-} B^{C}\right)_{s}^{+}$.

Proof. It remains only to prove c). Set $L_{s}^{ \pm}=\left(\Lambda^{-} B^{C}\right)_{s}^{ \pm}$. From (4.4.3) it follows (like in [15, Chap. II, Lemma 2.4]) that there are open subsets $U_{1}$ and $U_{2}$ contained in $L_{s}^{-}$and $L_{s}^{+}$ respectively, such that $U_{1} \cdot U_{2}$ is an open neighbourhood of $e$ in $\Lambda^{-} B^{C}$. Thus $L_{s}^{-} \cdot L_{s}^{+}=$ $\bigcup_{l \in L_{s}^{-}, r \in L_{s}^{+}} l U_{1} \cdot U_{2} r$ is open in $\Lambda^{-} B^{C}$. But $L_{s}^{-} \cdot L_{s}^{+}$is also closed in $\Lambda^{-} B^{C}$. To see this we consider a sequence

$$
b_{-}^{(n)} \cdot b_{+}^{(n)} \rightarrow b, \quad b_{-}^{(n)} \in L_{s}^{-}, b_{+}^{(n)} \in L_{s}^{+} .
$$

Then $s b_{-}^{(n)} s^{-1} \rightarrow s b s^{-1}$ converges in $\Lambda B^{C}$. But part c) of Lemma 4.3 shows

$$
s b_{-}^{(n)} s^{-1} \in \Lambda_{*}^{-} B^{C} \quad \text { and } \quad s b_{+}^{(n)} s^{-1} \in \Lambda^{+} B^{C} ;
$$

therefore $s b_{-}^{(n)}$ and $s b_{+}^{(n)}$ converge separately. Since $L_{s}^{-}$and $L_{s}^{+}$are closed, we have

$$
b_{-}^{(n)} \rightarrow b_{-} \in L_{s}^{-} \quad \text { and } \quad b_{+}^{(n)} \rightarrow b_{+} \in L_{s}^{+} .
$$

Hence $L_{s}^{-} \cdot L_{s}^{+}$is closed in $\Lambda B^{C}$. Since $B$ is simply connected, its universal complexification $B^{C}$ is also simply connected $[17,17.5]$. As a consequence, every loop in $B^{C}$ can be deformed into a point, i.e., $\Lambda B^{C}$ is path connected. Therefore the open and closed subset $L_{s}^{-} \cdot L_{s}^{+}$ coincides with $\Lambda B^{C}$. 
4.5. Under the assumptions made in Section 2.1, the Birkhoff decomposition for the loop group $\Lambda G^{C}$ is provided by

THEOREM. Any element $g \in \Lambda G^{C}$ can be written as

$$
g=g_{-} D g_{+},
$$

where $g_{ \pm} \in \Lambda^{ \pm} G^{C}, D=s b_{-}^{+} \in \Lambda^{d} G^{C}$, and

$$
\Lambda^{d} G^{C}=\bigcup_{s \in \Lambda^{d} H^{C}} s\left(\Lambda^{-} B^{C}\right)_{s}^{+}
$$

and

$$
\begin{aligned}
& \left(\Lambda^{-} B^{C}\right)_{s}^{-}=\left\{b \in \Lambda^{-} B^{C} \mid s b s^{-1} \in \Lambda^{-} B^{C}\right\}, \\
& \left(\Lambda^{-} B^{C}\right)_{s}^{+}=\left\{b \in \Lambda^{-} B^{C} \mid s b s^{-1} \in \Lambda_{*}^{+} B^{C}\right\} .
\end{aligned}
$$

The expression (4.5.1) will be called a Birkhoff decomposition of $g$. A representation of $g$ given in the form (4.5.6) satisfying (4.5.7) and (4.5.8) is unique and will be called canonical.

Proof. First we use the decomposition (3.2.1), which provides $g=h b \in \Lambda H^{C} \cdot \Lambda B^{C}$. Also, since $H^{C}$ is reductive, we can apply for $h \in \Lambda H^{C}$ the Birkhoff decomposition Theorem 4.4 of $\Lambda H^{C}$. Then $g=h b$ can be rewritten as

$$
g=h_{-} s h_{+} b=h_{-} s\left(h_{+} b{h_{+}}^{-1}\right) h_{+},
$$

where $s \in \Lambda^{d} H^{C} \subset \Lambda H^{C}$. But $h_{+} b h_{+}{ }^{-1} \in \Lambda B^{C}$, and hence by Lemma 4.3 it is decomposable, $h_{+} b h_{+}{ }^{-1}=b_{-} b_{+}$, with $b_{-} \in \Lambda_{*}^{-} B^{C}$. Then $g=h_{-} s \cdot b_{-} b_{+} \cdot h_{+}$and, using the splitting of Lemma 4.4 into the subgroups (4.5.3),

$$
\Lambda^{-} B^{C}=\left(\Lambda^{-} B^{C}\right)_{s}^{-} \cdot\left(\Lambda^{-} B^{C}\right)_{s}^{+},
$$

we obtain the decomposition

$$
g=\left(h_{-}\left(s b_{-}^{-} s^{-1}\right)\right) \cdot\left(s b_{-}^{+}\right) \cdot\left(b_{+} h_{+}\right)=g_{-} \cdot D \cdot g_{+} .
$$

So we have $\Lambda G^{C}=\Lambda^{-} G^{C} \cdot \Lambda^{d} G^{C} \cdot \Lambda^{+} G^{C}$. Moreover, by [22, Corollary 5 (c)] we can assume

$$
s^{-1} h_{-} s \in U_{-},
$$

and also have

$$
b_{-}^{-} b_{-}^{+} \in \Lambda_{*}^{-} B^{C} .
$$

In this case all factors occurring in (4.5.6) are uniquely determined by $g$. Assume that $g$ has two representations of the special form claimed, the second one being indicated with a "^ ". Then the described splitting procedure yields $h_{-} s h_{+}=\hat{h}_{-} \hat{s} \hat{h}_{+}$and $h_{+}^{-1} b_{-}^{-} b_{-}^{+} b_{+} h_{+}=$ $\hat{h}_{+}^{-1} \hat{b}_{-}^{-} \hat{b}_{-}^{+} \hat{b}_{+} \hat{h}_{+}$. Our assumption on $h_{-}$and $\hat{h}_{-}$implies in view of [22, Corollary 5 (c)] that $h_{-}=\hat{h}_{-}, s=\hat{s}$ and $h_{+}=\hat{h}_{+}$. As a consequence, $b_{-}^{-} b_{-}^{+} b_{+}=\hat{b}_{-}^{-} \hat{b}_{-}^{+} \hat{b}_{+}$. Since we have assumed that $b_{-}^{-} b_{-}^{+} \in \Lambda_{*}^{-} B^{C}$, we obtain by Lemma 4.3 and Lemma 4.4 that the corresponding factors are the same. 


\section{The "big cell" of the Birkhoff decomposition.}

5.1. We consider the "big cell" for the group $\Lambda G^{C}$,

$$
P_{G^{C}} \stackrel{\text { def }}{=} \Lambda^{-} G^{C} \cdot \Lambda^{+} G^{C} .
$$

Similarly, we define the big cell for the other loop groups like $\Lambda H^{C}$ and $\Lambda B^{C}$. For the elements in the big cell we have two types of splittings. On the one hand we have $g=g_{-} . g_{+}$ in $P_{G} c$, but also $g=h . b$ in view of (3.2.1). Comparing these two splittings, we have

Proposition. If $g=h b=g_{-} g_{+} \in P_{G}$, then the $\Lambda H^{C}$-component splits as $h=$ $h_{-} h_{+} \in P_{H^{c}}$, and in the decompositions (4.5.5) and (4.5.6) the element $s$ is the unity $e$ of $G^{C}$.

PROOF. From $g=g_{-} g_{+}$and (4.5.5) we obtain

$$
s\left(h_{+} b h_{+}^{-1}\right)=\left(h_{-}^{-1} g_{-}\right) \cdot\left(g_{+} h_{+}^{-1}\right) .
$$

Using the splittings (3.2.2) and (3.2.3) for the right two factors, we obtain

$$
s\left(h_{+} b h_{+}^{-1}\right)=\left(\tilde{h}_{-} \tilde{b}_{-}\right) \cdot\left(\tilde{h}_{+} \tilde{b}_{+}\right)=\tilde{h}_{-} \tilde{h}_{+}\left(\tilde{h}_{+}^{-1} \tilde{b}_{-} \tilde{h}_{+}\right) \tilde{b}_{+} \cdot
$$

But (2.1.4) implies that $s=\tilde{h}_{-} \tilde{h}_{+}$, and using the Birkhoff decomposition of $\Lambda H^{C}$ [23, Chap. 8.6], we obtain

$$
s=e .
$$

Then, using (5.1.2), $g=h_{-} h_{+} b$, and $g=h b$ implies $h=h_{-} h_{+} \in P_{H^{c}}$.

Lemma. Let $D=s \cdot b_{-}^{+} \in \Lambda^{d} G^{C}$. Then

$$
D=e \Leftrightarrow s=e \quad \text { and } \quad b_{-}^{+}=e \Rightarrow s=e .
$$

Proof. The first equivalence follows from (2.1.4). Assume now $s=e$. Then the definition of $\left(\Lambda^{-} B^{C}\right)_{s}^{+}$implies $b_{-}^{+}=e$.

The "big cell" $P_{G}$ is characterized by

Corollary. $g \in \Lambda G^{C}$ is in the big cell $P_{G} C \subset \Lambda G^{C}$ if and only if in the decomposition (4.5.6) one has $D=e$.

Proof. Let $g \in P_{G}$. Then $s=e$ and the Lemma yields $D=e$. The converse of the statement is trivial.

5.2. The elements of $P_{G} C$ lying in $\Lambda B^{C}$ split in $\Lambda B^{C}$, i.e., we have

Proposition. Let $b=g_{-} g_{+} \in P_{G^{C}} \cap \Lambda B^{C}$. Then $b \in P_{B}$, with $b_{-} \in \Lambda^{-} B^{C}, b_{+} \in$ $\Lambda^{+} B^{C}$.

PROOF. Using the decompositions (3.2.2) and (3.2.3), we obtain

$$
b=g_{-} g_{+}=\left(g_{-}^{H} g_{-}^{B}\right)\left(g_{+}^{H} g_{+}^{B}\right)=\left(g_{-}^{H} g_{+}^{H}\right)\left(g_{+}^{H-1} g_{-}^{B} g_{+}^{H}\right) g_{+}^{B},
$$

with $g_{-}^{H} \in \Lambda^{-} H^{C}$ and $g_{-}^{B} \in \Lambda^{-} B^{C}$. Then making use of (2.1.4) and identifying the extreme terms, we get $g_{-}^{H} g_{+}^{H}=e$, which implies that the two elements are inverse to each other and 
contained in $H$. Altogether we obtain $b=b_{-} b_{+}$with $b_{-}=g_{+}^{H-1} g_{-}^{B} g_{+}^{H} \in \Lambda^{-} B^{C}$ and $b_{+}=g_{+}^{B} \in \Lambda^{+} B^{C}$.

Lemma. Let $b=g_{-} g_{+} \in P_{G^{C}} \cap \Lambda B^{C}$. If $g_{-} \in \Lambda_{*}^{-} G^{C}$, then $b \in P_{B^{C}}$, with $b_{-}=$ $g_{-} \in \Lambda_{*}^{-} B^{C}, b_{+}=g_{+} \in \Lambda^{+} B^{C}$.

Proof. Using the decompositions (3.2.2) and (3.2.3), we obtain

$$
b=g_{-} g_{+}=\left(g_{-}^{H} g_{-}^{B}\right)\left(g_{+}^{H} g_{+}^{B}\right)=\left(g_{-}^{H} g_{+}^{H}\right)\left(g_{+}^{H-1} g_{-}^{B} g_{+}^{H}\right) g_{+}^{B} .
$$

Since $g_{-}^{H} \in \Lambda_{*}^{-} H^{C}$ and $g_{-}^{B} \in \Lambda_{*}^{-} B^{C}$, the relation $g_{-} g_{+}=e$ implies $g_{-}^{H}=g_{+}^{H}=e$, which proves the claim.

5.3. Based on this result, we can prove the

THEOREM. The "big cell" $P_{G^{c}}$ is open and dense in $\Lambda G^{C}$, and the mapping

$$
\Lambda^{-} G^{C} \times \Lambda^{+} G^{C} \rightarrow \Lambda G^{C}
$$

provides a surjective submersion onto the big cell.

Proof. The proof follows the one given in [23, Chap. 8] and [10, Chap. 2]. Let $\chi=$ $L^{2}\left(S^{1}, \boldsymbol{C}\right)=\chi_{-} \oplus \chi_{+}$, where

$$
\begin{aligned}
& \chi_{+}=\left\{\eta_{+} \in \chi \mid \eta_{+}=\sum_{n \geq 0} z_{n} \lambda^{n}, z_{n} \in \boldsymbol{C}\right\}, \\
& \chi_{-}=\left\{\eta_{-} \in \chi \mid \eta_{-}=\sum_{n<0} z_{n} \lambda^{n}, z_{n} \in \boldsymbol{C}\right\} .
\end{aligned}
$$

Let $\tau$ be the non-trivial holomorphic section of the dual of the determinant bundle (Det* ${ }^{*}, \pi r(\chi)$ ) (see, e.g., $[10,2.10]$ ). Then, using the faithful representation $G^{C} \rightarrow$ $S L(m, C)$, we see that $\Lambda G^{C}$ admits a natural representation $\Lambda G^{C} \rightarrow G L_{\text {res }}(\chi)$. Moreover, for the Grassmannian $\operatorname{Gr}(\chi)$ we obtain $\operatorname{Gr}(\chi)=G L_{\text {res }}(\chi) \cdot \chi_{+}$. Using (4.5.1) for $g=$ $g_{-} D g_{+}$, we have

$$
\Psi(g)=\tau\left(g_{-} \cdot D \cdot g_{+} \cdot \chi_{+}\right)=\tau\left(g_{-} D \cdot \chi+\right),
$$

and hence,

$$
\Psi(g) \neq 0 \Leftrightarrow \tau\left(D \cdot \chi_{+}\right) \neq 0 \Leftrightarrow D \in \Lambda^{-} S L(m, \boldsymbol{C}) \cdot \Lambda^{+} S L(m, \boldsymbol{C}),
$$

where we used that $\tau$ is invariant under left $\Lambda^{-} G^{C}$ translations (see, e.g., the proof of Lemma 2.4 in [10]). Also, from Corollary 5.1 we know $g \in P_{G} c \Leftrightarrow s=e \Leftrightarrow D=e$. Therefore $g \in P_{G^{C}}$ if and only if $\Psi \neq 0$. Since $\Psi$ is not the constant map with value $0, P=\Psi^{-1}\{\boldsymbol{C} \backslash$ $\{0\}\}=\Lambda G^{C} \backslash \Psi^{-1}\{0\}$ is open and dense in $\Lambda G^{C}$.

6. The Iwasawa decomposition. Let $G$ be a connected real Lie group which admits a faithful finite dimensional continuous representation.

6.1. First we consider the reductive subgroup $H$ of $G$ introduced in Chapter 2. We have a decomposition of the reductive subgroup $H$ in the form of a semisimple Lie group $S$ and a compact abelian Lie group $K, H=S \cdot K$. Also, we have $H^{C}=S^{C} \cdot K^{C}$. 
The generalized Iwasawa decomposition for reductive groups is provided by the following result of Kellersch [20, Chap. 4].

THEOREM. If $H$ is a connected reductive Lie group admitting a faithful finite dimensional representation, then

$$
\Lambda H^{C}=\Lambda H \cdot \Lambda^{m} H^{C} \cdot \Lambda^{+} H^{C},
$$

where $\Lambda^{m} H^{C}$ is a specific set of representatives for the double cosets in (6.1.1) given in [20]. For a brief description of $\Lambda^{m} H^{C}$, see the Appendix.

REMARK. Roughly speaking, the set $\Lambda^{m} H^{C}$ is as big as the set of all homomorphisms of $S^{1}$ into a maximal torus of $H^{C}$. More precisely, the elemnts of $\Lambda^{m} H^{C}$ consist of "twisted square roots" of such homomorphisms. For more on this see the Appendix. For examples see Section 9.

6.2. Next we consider the solvable normal subgroup $B$ of $G$ introduced in Chapter 2 and its subgroups $A$ and $N$. First we show

Lemma. $\quad \Lambda A^{C}=\Lambda A \cdot \Lambda^{+} A^{C}$.

PROOF. In view of (2.1.7) and (4.1.2) we consider the decomposition

$$
\Lambda \boldsymbol{C} \cong \Lambda \boldsymbol{R}+\Lambda^{+} \boldsymbol{C}
$$

provided by

$$
\sum_{k \in \boldsymbol{Z}} z_{k} \lambda^{k}=\sum_{k<0}\left(z_{k} \lambda^{k}+\bar{z}_{k} \bar{\lambda}^{k}\right)+\sum_{k \geq 0} z_{k} \lambda^{k}-\sum_{k<0} \bar{z}_{k} \bar{\lambda}^{k} \in \Lambda \boldsymbol{R}+\Lambda^{+} \boldsymbol{C},
$$

This implies the stated loop group decomposition

$$
\Lambda A^{C} \cong \Lambda A \cdot \Lambda^{+} A^{C} \cong(\Lambda R)^{p}+\left(\Lambda^{+} \boldsymbol{C}\right)^{p} .
$$

REMARK. This decomposition can be made unique, if one requires that the constant terms in $\Lambda^{+} \boldsymbol{C}$ are purely imaginary.

6.3. Also, for the nilpotent part $N$ of $B$ we have an Iwasawa decomposition

LEMMA. The nilpotent loops decompose as follows

$$
\Lambda N^{C}=\Lambda N \cdot \Lambda^{+} N^{C} .
$$

Proof. This proof is analogous to the one in Lemma 4.2. Using the same notation, every element $n \in N$ can be written in the form (4.2.3). Let $\tilde{n} \in \Lambda N^{C}$. We define inductively

$$
\tilde{n}^{(k+1)}=u_{k}^{-} \cdot \tilde{n}^{(k)} \cdot u_{k}^{+}, \quad k \geq 0, \quad \tilde{n}^{(0)}=\tilde{n},
$$

where

$$
u_{k}=\prod_{j=0}^{m_{k}} \exp \left(-\alpha_{k j}^{(k) *} \cdot b_{k j}\right), \quad u_{k}^{+}=\prod_{j=0}^{m_{k}} \exp \left(-\alpha_{k j}^{(k)+} \cdot b_{k j}\right),
$$


and where at each step $k \geq 0$, the coefficients $\alpha_{k j}^{(k) *}$ and $\alpha_{k j}^{(k)+}$ are provided by the decompositions

$$
\tilde{n}^{(k)}=\prod_{i \geq k} \prod_{j=0}^{m_{i}} \exp \left(\alpha_{i j}^{(k)} \cdot b_{i j}\right), \quad \alpha_{k j}^{(k)}=\alpha_{k j}^{(k) *}+\alpha_{k j}^{(k)+} \in \Lambda \boldsymbol{R}+\Lambda^{+} \boldsymbol{C} .
$$

After a finite number $m$ of steps we obtain $\tilde{n}^{(m)}=e \in \Lambda N^{C}$. Hence, denoting

$$
v_{k}=\left(u_{k}\right)^{-1} \in \Lambda N, \quad v_{k}^{+}=\left(u_{k}^{+}\right)^{-1} \in \Lambda^{+} N^{C}, \quad k \in\{0, \ldots, m-1\},
$$

we can decompose $\tilde{n}$ as

$$
\tilde{n}=\left(v_{0} \ldots v_{m-1}\right) \cdot\left(v_{m-1}^{+} \ldots v_{0}^{+}\right) \in \Lambda N \cdot \Lambda^{+} N^{C} .
$$

REMARK. This decomposition can be made unique, if one requires that in (6.3.4) the constant term in the $\Lambda^{+} \boldsymbol{C}$ part is purely imaginary.

6.4. Combining the results of 6.2 and 6.3 we finally show the following

Lemma. $\Lambda B^{C}=\Lambda B \cdot \Lambda^{+} B^{C}$.

Proof. Using (6.2.3) and (6.3.1), for $\tilde{b} \in \Lambda B^{C}$, we have

$$
\tilde{b}=\tilde{a} \tilde{n}=a a_{+} n n_{+}=\left(a \cdot\left(a_{+} n a_{+}^{-1}\right)\right) \cdot\left(a_{+} n_{+}\right) \in \Lambda A \cdot \Lambda N^{C} \cdot \Lambda^{+} B^{C},
$$

where we used $\Lambda N^{C} \triangleleft \Lambda B^{C}$ and the Wiener-Levi theorem for loops in $\Lambda B^{C} / \Lambda N^{C}$. Applying (6.3.1) to $a_{+} n a_{+}^{-1}$ implies the claim.

COROLlary. Using unique decompositions for $\Lambda A^{C}$ and $\Lambda N^{C}$, every $b \in \Lambda B^{C}$ has a unique decomposition of the form

$$
b=a n \cdot m_{+} a_{+} n_{+},
$$

where

$$
\begin{aligned}
& a \in \Lambda A, \quad n \in \Lambda N, \quad a_{+} \in \Lambda^{+} A^{C}, \quad m_{+}, n_{+} \in \Lambda^{+} N^{C}, \\
& a_{+}^{-1} b m_{+} a_{+} \in \Lambda N .
\end{aligned}
$$

Proof. The proof of the Lemma shows that every $b \in \Lambda B^{C}$ has a decomposition (6.4.1) satisfying (6.4.2) and (6.4.3). Assume we have two such decompositions. Then

$$
a a_{+} \cdot a_{+}^{-1} n m_{+} a_{+} n_{+}=\hat{a} \hat{a}_{+} \cdot \hat{a}_{+}^{-1} \hat{n} \hat{m}_{+} \hat{a}_{+} \hat{n}_{+} .
$$

Hence, in view of (2.1.2) we obtain $a a_{+}=\hat{a} \hat{a}_{+}$, whence, $a=\hat{a}$ and $a_{+}=\hat{a}_{+}$. Substituting this into (6.4.4), we derive

$$
n m_{+} a_{+} n_{+}=\hat{n} \hat{m}_{+} a_{+} \hat{n}_{+} .
$$

Therefore

$$
a_{+}^{-1}\left(n m_{+}\right) a_{+} \cdot n_{+}=a_{+}^{-1}\left(\hat{n} \hat{m}_{+}\right) a_{+} \cdot n_{+} .
$$


Because of (6.4.3), this implies $a_{+}^{-1}\left(n m_{+}\right) a_{+}=a_{+}^{-1}\left(\hat{n} \hat{m}_{+}\right) a_{+}$and $n_{+}=\hat{n}_{+}$. But then also $n=\hat{n}$ and $m_{+}=\hat{m}_{+}$.

6.5. Finally we obtain the following Iwasawa decomposition for an arbitrary untwisted group $\Lambda G^{C}$

THEOREM. Let $G$ be a connected real Lie group, which admits a finite-dimensional faithful representation. Then

$$
\Lambda G^{C}=\Lambda G \cdot \Lambda^{m} G^{C} \cdot \Lambda^{+} G^{C}
$$

is a disjoint union of double cosets indexed by the middle terms

$$
\Lambda^{m} G^{C}=\bigcup_{s \in \Lambda^{m} H^{C}}(\Lambda B)_{s}^{\#} \cdot s,
$$

where

$$
(\Lambda B)_{s}^{\#}=s \cdot \Lambda B \cdot s^{-1} .
$$

More precisely, every $g \in \Lambda G^{C}$ has a unique representation of the form

$$
g=h b \cdot w s b_{+} h_{+},
$$

where

$$
g=\tilde{h} \tilde{b}
$$

and

$$
\tilde{h}=h s h_{+}
$$

is the unique representation of $\tilde{h} \in \Lambda H^{C}=\Lambda H \cdot \Lambda^{m} H^{C} \cdot \Lambda^{+} H^{C}$ as described in the Appendix,

$$
\begin{aligned}
& \tilde{b}=\left(s h_{+}\right)^{-1} \cdot b w s b_{+} s^{-1} \cdot s h_{+}, \\
& b \in \Lambda B, \\
& w \in s \Lambda B s^{-1}, \quad b_{+} \in \Lambda^{+} B^{C}, \\
& w s b_{+} s^{-1} \in \Lambda^{+} B^{C} .
\end{aligned}
$$

Proof. Let $g \in \Lambda G^{C}$. Then (3.2.1) and Theorem 6.1 yield

$$
\tilde{g}=\tilde{h} \tilde{b}=h s h_{+} \tilde{b}=h\left[\left(s h_{+}\right) \tilde{b}\left(s h_{+}\right)^{-1}\right]\left(s h_{+}\right),
$$

where $\hat{h}=h s h_{+}$is the unique decomposition given in the Appendix. Since the term inside the square bracket is in $\Lambda B^{C} \triangleleft \Lambda G^{C}$, it can be split as in Lemma 6.4. This gives

$$
\tilde{g}=h \cdot b q_{+} \cdot s h_{+}=h b \cdot q_{+} s \cdot h_{+} .
$$

Since $\Lambda B^{C}=s \Lambda B s^{-1} \cdot s \Lambda^{+} B^{C} s$, we can decompose

$$
q_{+}=w l \text {, with } s^{-1} w s \in \Lambda B \quad \text { and } \quad s^{-1} l s=b_{+} \in \Lambda^{+} B^{C} .
$$

Thus $q_{+} s=w s \cdot s^{-1} l s=w s b_{+}$, and $g$ has the form (6.5.4). This, of course, implies (6.5.1). 
Assume now that $g$ has two representations of the form (6.5.4). We denote the second representation by the same letters, but use a superscript "^" in addition. Then (6.5.4) implies

$$
h s h_{+} \cdot\left(s h_{+}\right)^{-1}\left(b w s b_{+} s^{-1}\right) \cdot\left(s h_{+}\right)=\hat{h} \hat{s} \hat{h}_{+} \cdot\left(\hat{s} \hat{h}_{+}\right)^{-1}\left(\hat{b} \hat{w} \hat{s} \hat{b}_{+} \hat{s}^{-1}\right) \cdot\left(\hat{s} \hat{b}_{+}\right) .
$$

Therefore $h s h_{+}=\hat{h} \hat{s} \hat{h}_{+}$and, since this decomposition was chosen unique, we obtain $h=$ $\hat{h}, s=\hat{s}$ and $h_{+}=\hat{h}_{+}$. Substituting this into (6.5.14), we obtain

$$
b w s b_{+} s^{-1}=\hat{b} \hat{w} s \hat{b}_{+} s^{-1} .
$$

From (6.5.10) and (6.5.8) we derive now in view of Lemma 6.4

$$
b=\hat{b} \quad \text { and } \quad w s b_{+} s^{-1}=\hat{w} s \hat{b}_{+} s^{-1} .
$$

The last equation yields

$$
s^{-1} w s \cdot b_{+}=s^{-1} \hat{w} s \cdot \hat{b}_{+} .
$$

From (6.5.9) and Lemma 6.4 we thus obtain $b_{+}=\hat{b}_{+}$and $s^{-1} w s=s^{-1} \hat{w} s$, whence $w=$ $\hat{w}$.

\section{The "big cell" of the Iwasawa decomposition.}

7.1. In theorem 5.3 we have seen that one of the double cosets in the Birkhoff decomposition of $\Lambda G^{C}$, namely, the big cell $P_{G^{C}}$ is open and dense in $\Lambda G^{C}$, and that all other double cosets have a non-zero codimension in $\Lambda G^{C}$. It is therefore natural to investigate the double coset

$$
\tilde{P}_{G}=\Lambda G \cdot \Lambda^{+} G^{C}
$$

more closely. In the following, we justify the name "big cell" for $\tilde{P}_{G}$ by showing that under very weak assumptions it is open and dense in $\Lambda G^{C}$ and that under supplementary assumptions, it covers the whole loop-group $\Lambda G^{C}$.

LEMMA. $\tilde{P}_{G}$ is open in $\Lambda G^{C}$.

Proof. Let Lie $(T)$ denote the Lie algebra of a Banach Lie group $T$. With this notation we have

$$
\operatorname{Lie}\left(\Lambda G^{C}\right)=\operatorname{Lie}(\Lambda G)+\operatorname{Lie}\left(\Lambda^{+} G^{C}\right) .
$$

Therefore (as in [15, Chap. II, Lemma 2.4]) one can show that there exist open subsets $U \subset$ $\Lambda G$, with $e \in U$, and $U_{+} \subset \Lambda^{+} G^{C}$, with $e \in U_{+}$, such that $V=U \cdot U_{+}$is an open neighborhood of $e$ in $\Lambda G^{C}$. Note that the big cell $\tilde{P}_{G}$ is the union of the open sets

$$
\tilde{P}_{G}=\Lambda G \cdot \Lambda^{+} G^{C}=\bigcup_{g g_{+} \in \tilde{P}} g U \cdot U_{+} g_{+},
$$

and hence is open in $\Lambda G^{C}$.

7.2. For the untwisted loop groups $\Lambda G^{C}$, considered exclusively in this paper, we can extend a result of Kellersch [20, Theorem 4.58] from reductive groups to general groups. Note that Lemma 6.4 states $\tilde{P}_{B}=\Lambda B^{C}$. 
THEOREM. If the semisimple part of a maximal compact subgroup of $H^{C}$ is simply connected, then the big cell $\tilde{P}$ is open and dense in $\Lambda G^{C}$ and we have

$$
\tilde{P}_{G}=\tilde{P}_{H} \cdot \Lambda B^{C} \cong \tilde{P}_{H} \times \Lambda B^{C} .
$$

Proof. Let $\tilde{g}=g \cdot g_{+} \in \Lambda G \cdot \Lambda^{+} G^{C}$. Then $g=h b$ with $h \in \Lambda H$ and $b \in \Lambda B$, and $g_{+}=b_{+} \cdot h_{+}$with $b_{+} \in \Lambda^{+} B^{C}$ and $h_{+} \in \Lambda^{+} H^{C}$. Inserting this, we obtain $\tilde{g}=$ $h h_{+} \cdot h_{+}^{-1} b b_{+} h_{+}$, and since $\tilde{b}=h_{+}^{-1} b b_{+} h_{+} \in \Lambda B^{C}$, we see $\tilde{P}_{G} \subset \tilde{P}_{H} \cdot \Lambda B^{C}$.

Conversely, let $\tilde{g} \in \tilde{P}_{H} \cdot \Lambda B^{C}$. Then $\tilde{g}=h h_{+} \tilde{b}, h \in \Lambda H, h_{+} \in \Lambda^{+} H^{C}, \tilde{b} \in \Lambda B$ and $\tilde{g}$ can be written in the form

$$
\tilde{g}=h h_{+} \tilde{b} h_{+}^{-1} h_{+} .
$$

From Lemma 6.4 we know $h_{+} \tilde{b} h_{+}^{-1}=b b_{+}$with $b \in \Lambda B, b_{+} \in \Lambda^{+} B^{C}$, and $g \in \tilde{P}_{G}$ follows. This proves the first part of (7.2.1). From (2.1.4) the second part follows. But now the claim follows, since by [20, Theorem 4.58] $\tilde{P}_{H}$ is open and dense in $\Lambda H^{C}$ under our assumptions.

7.3. From [20, Theorem 4.48] we know that for a semisimple Lie group $S$ we have $\tilde{P}_{G}=\Lambda G^{C}$ if and only if $S$ is compact. This is at the heart of

Theorem. Let $G, H=S K$ and $S$ be as in Section 2.1 and Section 6.1. Then $\tilde{P}_{G}=$ $\Lambda G^{C}$ if and only if $S$ is compact.

Proof. (1) Assume first $S$ is compact. We prove the nontrivial inclusion $\Lambda G^{C} \subset$ $\tilde{P}_{G}=\Lambda G \cdot \Lambda^{+} G^{C}$. From [23] we know that $\Lambda S^{C}=\Lambda S \cdot \Lambda^{+} S^{C}$. Then Theorem 6.1 and the fact that $\Lambda K$ commutes with $\Lambda^{+} S^{C}$, imply $\Lambda H^{C}=\Lambda H \cdot \Lambda^{+} H^{C}$. Using (3.2.1) and Lemma 6.4, we obtain

$$
\Lambda G^{C}=\Lambda H^{C} \cdot \Lambda B^{C}=\Lambda H \cdot \Lambda^{+} H^{C} \cdot \Lambda B^{C}=\Lambda H \cdot \Lambda^{+} H^{C} \cdot \Lambda B \cdot \Lambda^{+} B^{C} .
$$

But every element

$$
g=h h_{+} b b_{+} \in \Lambda G^{C}=\Lambda H \cdot \Lambda^{+} H^{C} \cdot \Lambda B \cdot \Lambda^{+} B^{C}
$$

can be written in the form

$$
g=h \cdot h_{+} \cdot b \cdot b_{+}=h \cdot h_{+} b h_{+}^{-1} \cdot h_{+} b_{+}=h \cdot \tilde{b} \tilde{b}_{+} \cdot h_{+} b_{+},
$$

where we have used Lemma 6.4 again. This yields $g \in \tilde{P}_{G}$.

(2) Let now $\tilde{P}_{G}=\Lambda G^{C}$. Then the uniqueness statement of Theorem 6.5 shows that $\Lambda^{m} G^{C}$ only consists of $\{e\}$. In view of (6.5.11) and (6.1.1) Theorem, this yields that the Iwasawa decomposition of $\Lambda S^{C}$ consists of one double coset only. Therefore $S$ is compact [20, Theorem 4.48].

A somewhat different proof for the same result can be obtained using (7.2.1). 


\section{The Birkhoff decomposition-applications.}

8.1. In the case when $G$ is the nilpotent group

$$
G=\left\{\left(\begin{array}{cc}
1 & a \\
0 & 1
\end{array}\right) \mid a \in \boldsymbol{R}\right\},
$$

the decomposition is straightforward,

$$
\left(\begin{array}{cc}
1 & \sum_{k \in Z} a_{k} \lambda^{k} \\
0 & 1
\end{array}\right)=\left(\begin{array}{cc}
1 & \sum_{k<0} a_{k} \lambda^{k} \\
0 & 1
\end{array}\right) \cdot\left(\begin{array}{cc}
1 & \sum_{k \geq 0} a_{k} \lambda^{k} \\
0 & 1
\end{array}\right) \in \Lambda^{-} G^{C} \cdot \Lambda^{+} G^{C},
$$

8.2. For the group $G$ of upper triangular unipotent matrices

$$
G=\left\{\left(\left(\begin{array}{ccc}
1 & a & c \\
0 & 1 & b \\
0 & 0 & 1
\end{array}\right)\right) \mid a, b, c \in \boldsymbol{R}\right\}
$$

any loop $g \in \Lambda G$ can be decomposed

$$
g=\left(\begin{array}{ccc}
1 & a & c \\
0 & 1 & b \\
0 & 0 & 1
\end{array}\right)=\left(\begin{array}{ccc}
1 & a_{-} & c_{-} \\
0 & 1 & b_{-} \\
0 & 0 & 1
\end{array}\right) \cdot\left(\begin{array}{ccc}
1 & a_{+} & c_{+} \\
0 & 1 & b_{+} \\
0 & 0 & 1
\end{array}\right) \in \Lambda^{-} G^{C} \cdot \Lambda^{+} G^{C}
$$

where

$$
a=\sum_{k \in Z} a_{k} \lambda^{k}, b=\sum_{k \in Z} b_{k} \lambda^{k}, c=\sum_{k \in Z} c_{k} \lambda^{k},
$$

and the entries of the right factors are

$$
\left\{\begin{array}{l}
a_{+}=\sum_{k \geq 0} a_{k} \lambda^{k}, \quad a_{-}=\sum_{k<0} a_{k} \lambda^{k}, \\
b_{+}=\sum_{k \geq 0} b_{k} \lambda^{k}, \quad b_{-}=\sum_{k<0} b_{k} \lambda^{k}, \\
c_{+}=\sum_{k \geq 0}\left(c_{k}-\sum_{r<r(k)} a_{r} b_{k-r}\right) \lambda^{k}, \\
c_{-}=\sum_{k<0}\left(c_{k}-\sum_{r<r(k)} a_{r} b_{k-r}\right) \lambda^{k}, r(k)=\min \{0, k+1\} .
\end{array}\right.
$$

8.3. Let $G$ be the simple special linear Lie group

$$
G=S L(2, \boldsymbol{R})=\left\{\left(\begin{array}{ll}
a & b \\
c & d
\end{array}\right) \mid a, b, c, d \in \boldsymbol{R}, a d-b c=1\right\} .
$$

Then for $\Lambda G^{C}=\Lambda S L(2, C)$ we have the decomposition

$$
g=g_{-} \cdot D \cdot g_{+} \in \Lambda^{-} G^{C} \cdot \Lambda^{d} G^{C} \cdot \Lambda^{+} G^{C} .
$$

In general the Birkhoff decomposition has an arbitrary Weyl group element as middle term. In the case under consideration, however, the Weyl group has two parts, one just a permutation matrix and then the homomorphisms into a maximal torus. The permutation matrix is incorporated into one of the other two factors. Note, while in the first two sections we had 
examples, for which the splitting could be carried out by hand, in the present case the splitting is highly nontrivial and it is generally virtually impossible to carry out the splitting by hand.

8.4. Let $Q$ be a Lie group, and $\mathbf{q}=\operatorname{Lie}(Q)$ its Lie algebra. Consider

$$
G=T Q=\text { the tangent bundle of } Q .
$$

Then $G$ carries the structure of a Lie group (the tangent group) via the multiplication

$$
X_{a} * Y_{b}=\left(L_{a *} Y_{b}+R_{b *} X_{a}\right) \quad \text { for all } \quad X_{a} \in T_{a} Q, \quad Y_{b} \in T_{b} Q,
$$

where for all $a, x \in Q$, the maps $L_{a}(x)=a x$ and $R_{a}(x)=x a$ denote the left multiplication in $Q$ and the right multiplication in $Q$, respectively. Recall the trivialization of $T Q$ given by

$$
\tau: T Q \rightarrow Q \times \mathbf{q}, \quad \tau\left(X_{h}\right)=(h, X), \quad X=\theta\left(X_{h}\right) \quad \text { for all } \quad X_{h} \in T_{h} Q,
$$

where $\theta$ is the left Maurer-Cartan form of $G$,

$$
\theta\left(X_{h}\right)=L_{h^{-1} *} X_{h}, \quad \text { for all } X_{h} \in T_{h} Q .
$$

Identifying $T Q=Q \times \mathbf{q}$ via (8.4.3), the induced group operation on $Q \times \mathbf{q}$ is

$$
(h, X) \circ\left(h^{\prime}, X^{\prime}\right)=\left(h h^{\prime}, X^{\prime}+A d\left(h^{\prime}\right)^{-1} X\right),
$$

and the equation which provides the big cell decomposition is equivalent with

$$
h=h^{-} h^{+} \text {and } \quad X=X^{+}+A d\left(h^{+}\right)^{-1} X^{-} .
$$

In the general double-coset case, we have for an element $(h, X) \in \Lambda T Q^{C}$ the decomposition

$$
(h, X)=\left(h^{-}, X^{-}\right) \circ(\sigma, Z) \circ\left(h^{+}, X^{+}\right) \in \Lambda^{-} T Q^{C} \cdot \Lambda^{d} T Q^{C} \cdot \Lambda^{+} T Q^{C} .
$$

Using (8.4.5), this provides two equations: the first is the usual Birkhoff decomposition for $Q^{C}$-valued loops from Theorem 4.5

$$
h=h^{-} \cdot \sigma \cdot h^{+} \in \Lambda^{-} Q^{C} \cdot \Lambda^{d} Q^{C} \cdot \Lambda^{+} Q^{C},
$$

and the second relation

$$
X=X^{+}+A d\left(h^{+}\right)^{-1} Z+A d\left(\sigma h^{+}\right)^{-1} X^{-}
$$

provides the Lie algebra terms of the decomposition (8.4.7).

\section{The Iwasawa decomposition-applications.}

9.1. In the case, when $G$ is the nilpotent group defined in (8.1.1), the Iwasawa decomposition is straightforward,

$$
\left(\begin{array}{cc}
1 & \tilde{a} \\
0 & 1
\end{array}\right)=\left(\begin{array}{cc}
1 & a \\
0 & 1
\end{array}\right) \cdot\left(\begin{array}{cc}
1 & a^{+} \\
0 & 1
\end{array}\right) \in \Lambda G \cdot \Lambda^{+} G^{C},
$$

where $\tilde{a}=a+a^{+} \in \Lambda \boldsymbol{C}=\Lambda \boldsymbol{R}+\Lambda^{+} \boldsymbol{C}$ is performed as in (6.2.2). This is in accordance with Theorem 7.3, since for the semisimple subgroup $S$ contained in $G$ of 6.1 we have $S=\{e\}$. 
9.2. For the group $G$ of upper triangular unipotent matrices (8.2.1) any loop $g \in \Lambda G^{C}$ can be decomposed in the form

$$
\left(\begin{array}{ccc}
1 & \tilde{a} & \tilde{c} \\
0 & 1 & \tilde{b} \\
0 & 0 & 1
\end{array}\right)=\left(\begin{array}{ccc}
1 & a & a b+h \\
0 & 1 & b \\
0 & 0 & 1
\end{array}\right) \cdot\left(\begin{array}{ccc}
1 & a_{+} & h_{+} \\
0 & 1 & b_{+} \\
0 & 0 & 1
\end{array}\right) \in \Lambda G \cdot \Lambda^{+} G^{C},
$$

where

$$
\tilde{a}=a+a_{+}, \quad \tilde{b}=b+b_{+}, \quad \text { and } \quad \tilde{c}-a \cdot \tilde{b}=h+h_{+}
$$

are split as in (6.2.2). This is again in accordance with Theorem 7.3, since again $S=\{e\}$.

9.3. For the simple special linear Lie group $S L(2, \boldsymbol{R})$, the decomposition is much more less trivial. Any loop $\tilde{g} \in \Lambda S L(2, \boldsymbol{C})$ can be decomposed in the form [20, p. 122]

$$
\tilde{g}=g \cdot g_{m} \cdot g_{+} \in \Lambda G \cdot \Lambda^{m} G^{C} \cdot \Lambda^{+} G^{C},
$$

where the double cosets of the Iwasawa decomposition have the representatives $(\boldsymbol{N}=$ $\{0,1, \ldots\})$ :

$$
\begin{aligned}
\Lambda^{m} G^{C}= & \left\{\left(\begin{array}{cc}
\lambda^{p} & 0 \\
0 & \lambda^{-p}
\end{array}\right) \mid p \in N\right\} \bigcup \\
& \left\{\frac{1}{2}\left(\begin{array}{cc}
\lambda^{p}(\lambda+1) & i \lambda^{-p}\left(\lambda^{-1}-1\right) \\
i \lambda^{p}(\lambda-1) & \lambda^{-p}\left(\lambda^{-1}+1\right)
\end{array}\right) \mid p \in N\right\} .
\end{aligned}
$$

All the double cosets occurring in (9.3.2) are distinct. There is only one open double coset, the big cell. It is obtained for $p=0$ in the first set and is dense. All other double cosets have positive codimension in $\Lambda S L(2, \boldsymbol{C})$ [20].

9.4. For the tangent group in 8.4 , we have according to Theorem 6.5

$$
\Lambda T Q^{C}=\Lambda T Q \cdot \Lambda^{m} T Q^{C} \cdot \Lambda^{+} T Q^{C} .
$$

EXAMPLE 1. Let $Q$ be as in 6.1 and 9.1. Then for the maximal semisimple group $S$ contained in $Q$ we have $S=\{e\}$, and it is easy to show that also the maximal semisimple subgroups of $G=T Q$ is trivial. Therefore, by Theorem 7.3, the big cell covers the whole group $\Lambda T Q^{C}$. As a matter of fact the splitting is straightforward in this example:

$$
\begin{aligned}
\left(\left(\begin{array}{cc}
1 & \tilde{a} \\
0 & 1
\end{array}\right),\left(\begin{array}{cc}
0 & \tilde{X} \\
0 & 0
\end{array}\right)\right)= & \left(\left(\begin{array}{cc}
1 & a \\
0 & 1
\end{array}\right),\left(\begin{array}{cc}
0 & X \\
0 & 0
\end{array}\right)\right) \\
& \circ\left(\left(\begin{array}{cc}
1 & a^{+} \\
0 & 1
\end{array}\right),\left(\begin{array}{cc}
0 & X^{+} \\
0 & 0
\end{array}\right)\right)
\end{aligned}
$$

with the factors provided by the (6.2.2) type splittings $\tilde{a}=a+a^{+}, \quad \tilde{X}=X+X^{+}$.

EXAMPLE 2. Taking now $Q=S L(2, \boldsymbol{R})$, one can see that the middle term of the Lie algebra part of the decomposition vanishes and we have

$$
\begin{aligned}
(\tilde{g}, \tilde{X}) & =(g, X) \circ(\sigma, 0) \circ\left(g^{+}, X^{+}\right) \\
& \in(\Lambda Q \times \Lambda \mathbf{q}) \cdot\left(\Lambda^{m} Q^{C} \times\{0 \mathbf{q}\}\right) \cdot\left(\Lambda^{+} Q^{C} \times \Lambda^{+} \mathbf{q}^{C}\right) .
\end{aligned}
$$


Using (8.4.5) in (9.4.3), we find that $\tilde{g}=g . \sigma . g^{+}$is the usual Iwasawa decomposition (6.5.1) with $\sigma \in \Lambda^{m} Q^{C}=\Lambda^{m} S L(2, \boldsymbol{C})$ in (9.3.2), and that the terms of the Lie algebra part $X \in \Lambda \mathbf{q}$ and $X^{+} \in \Lambda^{+} \mathbf{q}^{C}$ are provided by the relation $\tilde{X}=A d\left(\sigma h^{+}\right)^{-1} X+X^{+}$or, equivalently,

$$
\operatorname{Ad}\left(\sigma h^{+}\right) \tilde{X}=X+\operatorname{Ad}\left(\sigma h^{+}\right) X^{+} .
$$

Appendix: A generalized Iwasawa decomposition in untwisted loop groups. This Appendix presents briefly the results of [20]; see also [9]. The main result of [20] is slightly sharpened and adjusted to the needs of this paper.

1. Let $\mathbf{g}$ be a finite dimensional, real semisimple Lie algebra and $\mathbf{g}^{C}$ its complexification. By $G^{C}$ we denote the simply connected and connected complex Lie group with Lie algebra $\mathbf{g}^{\boldsymbol{C}}$. We will always assume $G^{\boldsymbol{C}}$ to be a subgroup of some $G L(n, \boldsymbol{C})$ (see $[13,5.1]$ ). Let $\tau$ denote the (complex antilinear) complex conjugation in $\mathbf{g}^{C}$ with fixpoint algebra $\mathbf{g}$. Then $\tau$ has a (complex antilinear) lift to $G^{C}$ as an automorphism of order 2, also denoted by $\tau$. Let $G$ be the connected component of the identity element of $\operatorname{Fix}(\tau)=\left\{\mathbf{g} \in G^{C} \mid \tau(g)=g\right\}$.

Denoting as usual by $S^{1}$ the unit circle, we set

$$
\Lambda G^{C}=\left\{g: S^{1} \rightarrow \mathbf{g}^{C} \mid\|g\|<\infty\right\},
$$

where $g$ is a continuous function with Fourier expansion $g=\sum_{n \in Z} \lambda^{n} g_{n}$ and $\|g\|=$ $\sum_{n \in \mathbf{Z}}\left\|g_{n}\right\|<\infty$, where

$$
\left\|g_{n}\right\|=\max _{j \in\{1, \ldots, N\}} \sum_{i=1}^{N}\left|\left(g_{n}\right)_{i j}\right| .
$$

Then $\Lambda \mathbf{g}^{C}$ is a Banach Lie algebra.

Considering more generally

$$
\Lambda \operatorname{Mat}(N, \boldsymbol{C})=\left\{A: S^{1} \rightarrow \mathbf{g}^{C} \mid\|A\|<\infty\right\},
$$

where $\|A\|$ is defined as above, we see that $\Lambda \operatorname{Mat}(N, C)$ is an associative Banach algebra with identity $I$ and $\Lambda G^{C}$ is the connected component of the Banach group

$$
\Lambda G l(N, \boldsymbol{C})=(\Lambda \operatorname{Mat}(N, \boldsymbol{C}))^{*}=\{g \in \Lambda \operatorname{Mat}(N, \boldsymbol{C}) \mid g \text { is invertible }\},
$$

with Lie algebra $\operatorname{Lie} \Lambda G^{C}=\Lambda \mathbf{g}^{C}$.

2. The following Banach Lie subalgebras of $\Lambda \mathbf{g}^{C}$ will be of interest:

$$
\begin{aligned}
& \Lambda^{+} \mathbf{g}^{C}=\left\{h \in \Lambda \mathbf{g}^{C} \mid h_{n}=0 \text { for } n<0\right\}, \\
& \Lambda^{-} \mathbf{g}^{C}=\left\{h \in \Lambda \mathbf{g}^{C} \mid h_{n}=0 \text { for } n>0\right\} .
\end{aligned}
$$

It is easy to see that

$$
\Lambda^{+} \mathbf{g}^{C}+\Lambda^{-} \mathbf{g}^{C}=\Lambda \mathbf{g}^{C} .
$$

To make this a direct sum of vector spaces it suffices to make sure that for $\mathbf{g}_{0}^{+}$and $\mathbf{g}_{0}^{-}$, the $\lambda^{0}$-coefficients in $\Lambda^{+} \mathbf{g}^{C}$ and $\Lambda^{-} \mathbf{g}^{C}$ respectively, we have

$$
\mathbf{g}_{0}^{+}+\mathbf{g}_{0}^{-}=\mathbf{g}^{C}, \mathbf{g}_{0}^{+} \cap \mathbf{g}_{0}^{-}=\{0\} .
$$


For our purposes it will also be important to extend the conjugation $\tau$ to $\Lambda \mathbf{g}^{C}$ :

$$
(\tau g)(\lambda)=\tau(g(\lambda)), \quad \lambda \in S^{1} .
$$

Then we set

$$
\Lambda \mathbf{g}=\left\{g \in \Lambda \mathbf{g}^{C} \mid \tau g=g\right\} .
$$

Note. The coefficients in $\Lambda \mathbf{g}$ of $\lambda^{0}$ satisfy $\tau g_{0}=g_{0}$, i.e., $g_{0} \in \mathbf{g}=\operatorname{Fix}(\tau)$. It is easy to verify that $\Lambda \mathrm{g}$ is a (real) Banach subalgebra of $\Lambda \mathbf{g}^{C}$ and that we have

$$
\Lambda \mathbf{g}+\Lambda^{+} \mathbf{g}^{C}=\Lambda \mathbf{g}^{C} .
$$

To make this decomposition of $\Lambda \mathbf{g}^{C}$ unique, it suffices to find a subalgebra $\mathbf{s}_{0}^{+}$of $\mathbf{g}_{0}^{+}$such that

$$
\mathbf{g}+\mathbf{s}_{0}^{+}=\mathbf{g}^{C}, \mathbf{g} \cap \mathbf{s}_{0}^{+}=\{0\} .
$$

In $[20,1.77]$ it has been shown that such a (real, solvable) subalgebra $\mathbf{s}_{0}^{+}$can be found. Thus, on the Lie algebra level there exists a unique Iwasawa decomposition.

3. Finally, for our purposes it will be convenient to decompose $\Lambda^{+} \mathbf{g}^{C}$ and $\Lambda^{-} \mathbf{g}^{C}$ in a simple fashion: denoting by $\mathbf{c}$ the diagonal elements of $\mathbf{g}^{C}$, we set

$$
\begin{aligned}
& \mathbf{b}_{+}=\mathbf{c}+\mathbf{u}_{+}, \\
& \mathbf{b}_{-}=\mathbf{c}+\mathbf{u}_{-},
\end{aligned}
$$

where $\left(\mathbf{u}_{+}\right)_{0}$ and $\left(\mathbf{u}_{-}\right)_{0}$ consist of strictly upper and strictly lower triangular matrices, respectively.

Let $U_{ \pm}$denote the connected subgroup of $\Lambda^{ \pm} G^{C}$ with Lie algebra Lie $U_{ \pm}=\mathbf{u}_{ \pm}$. Then $U_{ \pm}$are simply connected and connected Banach subgroups of $\Lambda G^{C}$.

If $C$ denotes the connected subgroup of $G^{C}$ with Lie algebra $\mathbf{c}$, then $C$ and $B_{ \pm}=C U_{ \pm}$ are connected Banach Lie subgroups of $\Lambda G^{C}$.

By $N$ we denote the normalizer of $C$ in $\Lambda^{\mathrm{pol}} G^{C}$, the elements of $\Lambda G^{C}$ which are Laurent polynomials. Then $W=N / C$ is called the Weyl group of $\Lambda G^{C}$ (relative to $C$ ).

One can show that $N$ can be written in the form

$$
N=\stackrel{\circ}{N} \Pi \text {, }
$$

where $\stackrel{\circ}{N}$ denotes the normalizer of $C$ in $G^{C}$ and $\Pi$ denotes the group of all homomorphisms from $S^{1}$ to $T$, where $T$ is a maximal torus in $U$, and $U$ is a compact real form of $G^{C}$, and $(\text { Lie } T)^{C}=\mathbf{c}$.

Using the notation introduced above, the Birkhoff decomposition of $\Lambda G^{C}$ is given by

$$
\Lambda G^{C}=U_{-} N U_{+}=\bigcup_{w \in W} B_{-} w B_{+}=\bigcup_{w \in W} U_{-} w C U_{+},
$$

where $w$ denotes simultaneously an element of the Weyl group and a representative of $w$ in $N \subset \Lambda G^{C}$. This union is disjoint. For $w \in W$ we have

$$
\begin{aligned}
& U_{+}=\left(U_{+} \cap w B_{+} w^{-1}\right)\left(U_{+} \cap w B_{-} w^{-1}\right), \\
& U_{-} \cap w U_{+} w^{-1}=U_{-} \cap C w U_{+} w^{-1}
\end{aligned}
$$


and several similar relations.

4. Using the notation of the previous section, we can find roots of $\mathbf{g}^{C}$ and of $\Lambda \mathbf{g}^{C}$ such that $\mathbf{u}_{ \pm}$is spanned by the positive and negative roots of $\Lambda G^{C}$ respectively, with Cartan algebra

Then $U_{+}=\stackrel{\circ}{U}_{+} U_{+}^{\lambda}$, where $\stackrel{\circ}{U}_{+}=U_{+} \cap G^{C}$ and $U_{+}^{\lambda}=I+O(\lambda)$. Then for the complex conjugation $\tau$ we have $\tau\left(U_{+}^{\lambda}\right)=U_{-}^{\lambda}$. Moreover, there exists some $w_{0} \in \stackrel{\circ}{W}$, the Weyl group of $G^{C}$ relative to $\mathbf{c}$ such that

$$
\tau\left(U_{+}\right)=w_{0}^{-1} U_{-} w_{0} .
$$

Then we have the modified Birkhoff decomposition

$$
\Lambda G^{C}=\tau\left(U_{+}\right) N U_{+}=\bigcup_{w \in W} \tau\left(U_{+}\right) w C U_{+} .
$$

This union is disjoint. The representation of $y \in \Lambda G^{C}$ as an element in $\tau\left(U_{+}\right) w C U_{+}$can be made unique by choosing $s_{+}, r_{+} \in U_{+}$and $n \in N$ such that

$$
y=\tau\left(s_{+}\right) n r_{+} \quad \text { and } n r_{+} n^{-1} \in \tau\left(U_{-}\right) .
$$

Such choices can always be made.

5. In general terms, the generalized Iwasawa decomposition is the set of double cosets relative to the action of $\Lambda G \times \Lambda^{+} G^{C}$ on $\Lambda G^{C}$ given by

$$
\left(h, v_{+}\right) \cdot g=h g v_{+}^{-1} \text {. }
$$

Thus

$$
\Lambda G^{C}=\bigcup_{x \in I} \Lambda G \cdot x \cdot \Lambda^{+} G^{C},
$$

where $I$ parametrizes the double cosets and $x$ is $a$ representative for each coset.

The final goal is to make this much more explicit. To this end we start with $g \in \Lambda G^{C}$ and consider $x=\tau(g)^{-1} g$. Then $x$ is an element of the set of " $\tau$-symmetric elements"

$$
\mathcal{T}=\left\{p \in \Lambda G^{C} \mid \tau(p)=p^{-1}\right\} .
$$

Note

$$
\left\{\tau(g)^{-1} g \mid g \in \Lambda G^{C}\right\} \subset \mathcal{T}
$$

and the inclusion is (in general, certainly) strict.

Using the modified Birkhoff decomposition, one finds unique $n \in N, r_{+}, v_{+} \in U_{+}$such that

$$
\tau\left(r_{+}\right)^{-1} x r_{+}=n v_{+}, \quad \tau(n)=n^{-1}, \quad n v_{+} n^{-1}=\tau\left(v_{+}\right)^{-1} .
$$

In particular, $n v_{+}$is splittable. One considers the connected Lie group

$$
U_{++}=U_{+} \cap\left(w_{0} n\right)^{-1} U_{-}\left(w_{0} n\right) .
$$

Then there exists a unique $y=\exp X$ with

$$
X \in \operatorname{Lie} U_{++}, \quad d \Phi(X)=-X,
$$


where $\Phi(g)=\tau\left(n g n^{-1}\right)$ such that $\tau(y)^{-1} n v_{+} y=n$. Note that this implies that $n$ is splittable as well.

6. The next step is the splitting of $n \in N$. First we note that every $n \in N$ can be written uniquely in the form

$$
n=q \cdot \exp \left(H_{-}\right) \cdot t,
$$

where $t \in \Pi, H_{-} \in \mathbf{c}_{\boldsymbol{R}}$ and $q \in U, q \mathbf{t} q^{-1} \subset \mathbf{t}$, where $\mathbf{t}=$ Lie $T$. In our case we also know $\tau(n)=n^{-1}$. This implies

$$
\tau(q)=q^{-1}, \quad t=\tau\left(q t^{-1} q^{-1}\right)
$$

and $H_{-}=-\tau\left(q H_{-} q^{-1}\right)$. Setting $y_{0}=\exp \left(-\frac{1}{2} H_{-}\right)$, we obtain

$$
\tau\left(y_{0}\right)^{-1} n y_{0}=q \cdot t \text {. }
$$

Of course, $q t$ is again splittable. In [20], [9] it has been explained how to split elements of this type and that there exist only countably many double cosets.

Let $\Lambda^{m} G^{C}$ denote the set of $s$ such that $\tau(s)^{-1} s=q t$ for $q t$ in a set of representatives of the double cosets. Then altogether we have shown:

THEOREM (Generalized Iwasawa decomposition).

(1) $\Lambda G^{C}=\bigcup_{s \in \Lambda^{m} G^{C}} \Lambda G \cdot s \cdot U^{+}$. This union is disjoint.

(2) Every $g \in \Lambda G^{C}$ has a unique representation of the form

$$
g=h . s \quad y_{0} \text { y } r_{+},
$$

where

(i) $h \in \Lambda G$,

(ii) $\tau(s)^{-1} s=q t \in U \cap \stackrel{\circ}{N} \cdot \Pi$,

(iii) $\tau\left(s y_{0}\right)^{-1} s y_{0}=q \exp H_{-} t=n \in N, y_{0}=\exp \left(-\frac{1}{2} H_{-}\right), H_{-} \in \mathbf{c}_{\boldsymbol{R}}$,

(iv) $\tau\left(s y_{0} y\right)^{-1} s y_{0} y=n v_{+}, n v_{+} n^{-1}=\tau\left(v_{+}\right)^{-1}$, $y=\exp X \in U^{+} \cap\left(w_{0} n\right)^{-1} U_{-}\left(w_{0} n\right), y^{2}=v_{+}$, $d \Phi(X)=-X, \Phi(p)=\tau\left(n p n^{-1}\right)$,

(v) $\tau(g)^{-1} g=\tau\left(r_{+}\right)^{-1} \cdot n v_{+} \cdot r_{+}, r_{+} \in U_{+}, n r_{+} n^{-1} \in \tau\left(U_{-}\right)$.

PROOF. The existence of such a decomposition has been shown in [20], [9]. Let

$$
g=h s y_{0} y r_{+}=\tilde{h} \tilde{s} \tilde{y}_{0} \tilde{y} \tilde{r}_{+}
$$

be two decompositions as in (2). Then

$$
\tau(g)^{-1} g=\tau\left(r_{+}\right)^{-1} n v_{+} r_{+}=\tau\left(\tilde{r}_{+}\right)^{-1} \tilde{n} \tilde{v}_{+} \tilde{r}_{+} .
$$

Since $n v_{+}=\tau\left(v_{+}\right)^{-1} n$ and $\tilde{n} \tilde{v}_{+}=\tau\left(\tilde{v}_{+}\right)^{-1} \tilde{n}$,

$$
\tau\left(v_{+} r_{+}\right)^{-1} n r_{+}=\tau\left(\tilde{v}_{+} \tilde{r}_{+}\right)^{-1} \tilde{n} \tilde{r}_{+} .
$$

Since also $n r_{+} n^{-1} \in \tau\left(U_{-}\right)$and $\tilde{n} \tilde{r}_{+} \tilde{n}^{-1} \in \tau\left(U_{-}\right)$, the uniqueness of the generalized Birkhoff decomposition (see A.4.) implies $r_{+}=\tilde{r}_{+}, n=\tilde{n}$ and $v_{+} r_{+}=\tilde{v}_{+} \tilde{r}_{+}$, whence also $v_{+}=\tilde{v}_{+}$. 
Next, since the group

$$
U^{+} \cap\left(w_{0} n\right)^{-1} \cdot U_{-} \cdot\left(w_{0} n\right)
$$

is nilpotent, connected and simply connected, the equations

$$
y^{2}=v_{+}, \quad y=\exp X
$$

define $y$ uniquely from $v_{+}=\exp (+2 X)$. Moreover,

$$
\begin{aligned}
\tau(y)^{-1} n y & =\exp (-\tau X) \cdot n \cdot \exp (X)=n \cdot \tau\left(n \exp (-X) n^{-1}\right) \exp (X) \\
& =n \Phi(\exp (-X)) \cdot \exp (X)=n \exp X \cdot \exp X=n v_{+},
\end{aligned}
$$

where we have used $\tau(n)=n^{-1}$. Thus $\tau\left(s y_{0}\right)^{-1} s y_{0}=n=q \cdot \exp H_{-} \cdot t$. Note that $y_{0}=$ $\exp \left(-\frac{1}{2} H_{-}\right)$defines $y_{0}$ uniquely. Altogether, $v_{+}, y$ and $y_{0}$ have been determined uniquely. But $\tau(s)^{-1} s=q t$ and by definition $s$ is a uniquely chosen representative for the double cosets. Therefore also $s=\tilde{s}$ and $h=\tilde{h}$ follows.

\section{REFERENCES}

[ 1 ] V. BALAN, On the DPW method for the tangent group, Proceedings of the conference of differential geometry, Lie algebras and global analysis, Thessaloniki 1998, Geometry Balkan Press, Bucharest 2000, 1-9.

[ 2 ] V. BALAN AND J. DoRFMEISTER, The DPW method for harmonic maps from Riemann surfaces to general Lie groups, Balkan J. Geom. Appl. 5(2000), 7-37.

[3] V. BALAN AND J. DORFMEISTER, DPW for generalized symmetric spaces, in preparation.

[ 4 ] R. W. Brockett And H. J. Sussmann, Tangent bundles of homogeneous spaces are homogeneous spaces, Proc. Amer. Math. Soc. 35(1972), 550-551.

[ 5 ] N. Bourbaki, Lie Groups and Lie Algebras, Elements of Mathematics, Springer, Berlin, New York, 1989.

[6] L. BUNGART, On analytic fiber bundles, Topology 7(1967), 55-68.

[ 7 J. DORFMEISTER AND U. EITNER, Weierstrass type representation for affine spheres, submitted.

[ 8 ] J. Dorfmeister, H. Gradl and J. SzMigielski, Systems of PDE's obtained from factorization in loop groups, Acta Appl. Math. 53(1998), 1-58.

[ 9 ] J. Dorfmeister And P. Kellersch, A generalized Iwasawa decomposition in untwisted loop groups, in preparation.

[10] J. DorfmeISTER, F. PEDIT AND H. WU, Weierstrass type representations of harmonic maps into symmetric spaces, Comm. Anal. Geom. 6(1998), 633-668.

[11] J. Dorfmeister AND H. WU, Constant mean curvature surfaces and loop groups, J. Reine Angew. Math 440 (1993), 43-76.

[12] I. C. Gohberg, A factorization problem in normed rings, functions of isometric and symmetric operators and singular integral equations, Russian Math. Surveys 19(1964), 63-114.

[13] R. GoOdman AND N. WALLACH, Structure and unitary cocycle representations of loop groups and the group of diffeomorphisms of the circle, J. Reine Angew. Math. 347(1984), 69-133.

[14] F. HeLEIN, Willmore immersions and loop groups, J. Differential Geom. 50(1998), 331-385.

[15] S. Helgason, Differential Geometry, Lie Groups and Symmetric Spaces, Academic Press, New York, San Francisco, London, 1978.

[16] J. Hilgert AND K.-H. NEEB, Lie Semigroups and their Applications, Lecture Notes in Math. 1552, Springer, Berlin, 1993.

[17] G. Hochschild, The Structure of Lie Groups, Holden-Day, Inc., San Francisco, London, Amsterdam, 1965

[18] V. KAC, Infinite Dimensional Lie Algebras, Birkhüser, 1983.

[19] Y. Katznelson, An Introduction to Harmonic Analysis, Dover Publ. Inc., New York, 1976.

[20] P. KelLERSCH, Eine Verallgemeinerung der Iwasawa Zerlegung in Loop Gruppen, Dissertation, TU-Munich, 1999.

[21] A. L. Onishchik And E. B. Vinberg, Lie Groups and Algebraic Groups, Springer-Verlag, 1990. 
[22] D. H. Peterson And V. Kac, Infinite flag varieties and conjugacy theorems, Proc. Natl. Acad. Sci. USA 80(1983), 1778-1782.

[23] A. N. Pressley And G. Segal, Loop Groups, Oxford Math. Monographs, Clarendon Press, Oxford, 1986.

[24] K. UhlenBeCK, Harmonic maps into Lie groups (classical solutions of the chiral model), J. Differential Geom. 30(1989), 1-50

[25] V. S. VARADARAJAN, Lie Groups, Lie Algebras and Their Representations, Prentice Hall, 1974.

[26] K. YAno And S. Ishihara, Tangent and Cotangent Bundles, Marcel Dekker, 1973.

DEPARTMENT OF MATHEMATICS I

UNIVERSITY POLITEHNICA OF BUCHAREST

SPLAIUL INDEPENDENŢEI 313

RO-77206, BUCHAREST

ROMANIA

E-mail address: vbalan@mathem.pub.ro
DePartment OF MATHEMATICS

TU-MUENCHEN, ARCISTRASSE 123

D-80333 MuENCHEN

GERMANY

E-mail address: dorfm@ma.tum.de 